\title{
Characterization of a
} Pyrethroid-Degrading Pseudomonas fulva Strain P31 and Biochemical Degradation Pathway of D-Phenothrin

\begin{abstract}
Jingjing Yang ${ }^{1 \dagger}$, Yanmei Feng't, Hui Zhan', Jie Liu'², Fang Yang ${ }^{1}$, Kaiyang Zhang', Lianhui Zhang ${ }^{1}$ and Shaohua Chen ${ }^{1 *}$

${ }^{1}$ State Key Laboratory for Conservation and Utilization of Subtropical Agro-bioresources, Guangdong Province Key Laboratory of Microbial Signals and Disease Control, Integrative Microbiology Research Centre, South China Agricultural University, Guangzhou, China, ${ }^{2}$ Laboratory of Insect Toxicology, and Key Laboratory of Pesticide and Chemical Biology, Ministry of Education, South China Agricultural University, Guangzhou, China
\end{abstract}

\section{OPEN ACCESS}

Edited by:

Sabine Kleinsteuber,

Helmholtz-Zentrum für

Umweltforschung (UFZ), Germany

Reviewed by:

Maria Fátima Carvalho,

Centro Interdisciplinar de Pesquisa

Marine e Ambiental (CIIMAR),

Portugal

Fernando Govantes,

Universidad Pablo de Olavide, Spain

*Correspondence:

Shaohua Chen

shchen@scau.edu.cn

tThese authors have contributed equally to this work.

Specialty section:

This article was submitted to Microbiotechnology, Ecotoxicology and Bioremediation,

a section of the journal

Frontiers in Microbiology

Received: 05 February 2018 Accepted: 30 April 2018

Published: 16 May 2018

Citation:

Yang J, Feng $Y$, Zhan H, Liu J,

Yang $F$, Zhang $K$, Zhang $L$ and Chen S (2018) Characterization of a

Pyrethroid-Degrading Pseudomonas fulva Strain P31 and Biochemical

Degradation Pathway of D-Phenothrin.

Front. Microbiol. 9:1003.

doi: 10.3389/fmicb.2018.01003
D-phenothrin is one of the most popular pyrethroid insecticides for its broad spectrum and high insecticidal activity. However, continuous use of D-phenothrin has resulted in serious environmental contamination and raised public concern about its impact on human health. Biodegradation of D-phenothrin has never been investigated and its metabolic behaviors remain unknown. Here, a novel bacterial strain P31 was isolated from active sludge, which completely degraded (100\%) D-phenothrin at $50 \mathrm{mg} \cdot \mathrm{L}^{-1}$ in $72 \mathrm{~h}$. Based on the morphology, 16S rRNA gene and Biolog tests, the strain was identified as Pseudomonas fulva. Biodegradation conditions were optimized as $29.5^{\circ} \mathrm{C}$ and $\mathrm{pH} 7.3$ by utilizing response surface methodology. Strain P31 depicted high tolerance and strong D-phenothrin degradation ability through hydrolysis pathway. Strain P31 degraded D-phenothrin at inhibition constant $\left(K_{\mathrm{i}}\right)$ of $482.1673 \mathrm{mg} \cdot \mathrm{L}^{-1}$ and maximum specific degradation constant $\left(q_{\max }\right)$ of $0.0455 \mathrm{~h}^{-1}$ whereas critical inhibitor concentration remained as $41.1189 \mathrm{mg} \cdot \mathrm{L}^{-1}$. The 3-Phenoxybenzaldehyde and 1,2-benzenedicarboxylic butyl dacyl ester were identified as the major intermediate metabolites of D-phenothrin degradation pathway through high-performance liquid chromatography and gas chromatography-mass spectrometry. Bioaugmentation of D-phenothrin-contaminated soils with strain P31 dramatically enhanced its degradation, and over $75 \%$ of D-phenothrin was removed from soils within 10 days. Moreover, the strain illustrated a remarkable capacity to degrade other synthetic pyrethroids, including permethrin, cyhalothrin, $\beta$-cypermethrin, deltamethrin, fenpropathrin, and bifenthrin, exhibiting great potential in bioremediation of pyrethroid-contaminated environment.

Keywords: bioremediation, D-phenothrin, Pseudomonas fulva, metabolites, degradation pathway

\section{INTRODUCTION}

D-phenothrin [(1R-trans)-2,2-dimethyl-3-(2-methyl-1-propenyl)-(3-phenoxyphenyl) methyl ester] is a type I synthetic pyrethroid (SP) insecticide which contains a cyclopropanecarboxylic acid group linked to an aromatic alcohol through a central ester bond (Supplementary Figure 1). D-phenothrin is widely used to control insects related to public health including mosquitoes, ticks, 
mites, human head lice and scabies, as well as insect pests of animals and agricultural crops (Scott et al., 2015). SP insecticides are analogs of pyrethrins found in Chrysanthemum cinerariaefolium flowers (Cycoń and Piotrowska-Seget, 2016). Applications of SPs have significantly increased over the past two decades because of their ability to paralyze the target insects and low mammalian toxicity as compared to organochlorines and organophosphates (Scott et al., 2015). Half-life of pyrethroids is usually less than 600 days and are generally considered safe for humans and animals (Laskowski, 2002), but pyrethroid residues are frequently found in groundwater, sediments, foods, and mammals (Delgado-Moreno et al., 2011; Kuivila et al., 2012; Markle et al., 2014). Pyrethroid bioaccumulation was first reported in wild river fish in Iberian river basins of Spain (Corcellas et al., 2015), and in 100\% honeybee wax samples (García et al., 2017). Pyrethroid intoxication to endocrine activity causing estrogenic or antiestrogenic effects and immune responses in fish and mammals was also reported (Ansari et al., 2011; Saillenfait et al., 2015; Brander et al., 2016). In addition, D-phenothrin exposure to humans and mammals can lead to DNA damage in vitro (Nagy et al., 2014; Atmaca and Aksoy, 2015). Such serious environmental contaminations of SPs are not only effecting the wild life but human population as well. This situation demands for the advanced remediating strategies to tackle pyrethroid-polluted environments.

To remove pesticide residues from the environment, several remediation technologies such as photodecomposition, fenton degradation, ozonation, adsorption, incineration, and biodegradation have been developed (Arora et al., 2017; Cycoń et al., 2017; Morillo and Villaverde, 2017; Zhan et al., 2018a). Recently, bioremediation through bioaugmentation and/or biostimulation, has emerged as the most cost-effective and eco-friendly approach to breakdown pesticides in soils (Gao et al., 2012; Cycoń and Piotrowska-Seget, 2016). Different pyrethroid-degrading strains, such as Sphingobium sp. JZ-1 (Wang et al., 2009), Stenotrophomonas sp. ZS-S-01 (Chen et al., 2011d), Ochrobactrum anthropic YZ-1 (Zhai et al., 2012), Brevibacterium aureum DG-12 (Chen et al., 2013), Serratia marcescens (Cycoń et al., 2014), Bacillus thuringiensis ZS-19 (Chen et al., 2015), Pseudomonas aeruginosa GF31 (Tang et al., 2017), and Acinetobacter baumannii ZH-14 (Zhan et al., 2018b) have already been isolated from contaminated soils. However, microbial degradation of D-phenothrin and its pathway has never been investigated.

The objectives of this study were: (1) to investigate the microbial degradation of D-phenothrin and its biochemical degradation pathway; (2) to determine the biodegradation kinetics of D-phenothrin; and (3) to evaluate the potentials of isolate for bioremediation of D-phenothrin-contaminated environment.

\section{MATERIALS AND METHODS}

\section{Chemicals and Media}

D-phenothrin (98\% purity) was obtained from Jiangsu Yangnong Chemical Group Co., Ltd., China. Technical-grade permethrin, cyhalothrin, $\beta$-cypermethrin, deltamethrin, fenpropathrin, and bifenthrin were purchased from Sigma-Aldrich, United States and high-performance liquid chromatography (HPLC)-grade acetonitrile was purchased from Fisher Scientific, United States. All other chemicals and solvents were of analytical grade. SPs were dissolved in dimethyl sulfoxide (DMSO) or acetone at a stock concentration of $10 \mathrm{~g} \cdot \mathrm{L}^{-1}$, and stored in dark bottles at $4^{\circ} \mathrm{C}$.

Mineral salt medium (MSM) containing $\left(\mathrm{g} \cdot \mathrm{L}^{-1}\right)\left(\mathrm{NH}_{4}\right)_{2} \mathrm{SO}_{4}$, 2; $\mathrm{MgSO}_{4} \cdot 7 \mathrm{H}_{2} \mathrm{O}, 0.2 ; \mathrm{CaCl}_{2} \cdot 2 \mathrm{H}_{2} \mathrm{O}, 0.01 ; \mathrm{FeSO}_{4} \cdot 7 \mathrm{H}_{2} \mathrm{O}, 0.001$; $\mathrm{Na}_{2} \mathrm{HPO}_{4} \cdot 12 \mathrm{H}_{2} \mathrm{O}, 1.5$; and $\mathrm{KH}_{2} \mathrm{PO}_{4}, 1.5$; and Luria-Bertani (LB) medium containing $\left(\mathrm{g} \cdot \mathrm{L}^{-1}\right)$ tryptone, 10 ; yeast extract, 5; and $\mathrm{NaCl}, 10$ were used in this study. Both culture media were adjusted to $\mathrm{pH} 7.3$ and sterilized at $121^{\circ} \mathrm{C}$ for $20 \mathrm{~min}$.

\section{Isolation of D-Phenothrin-Degrading Bacterial Strains}

Active sludge from an aerobic pyrethroid-manufacturing wastewater treatment system in Zhongshan, China, was used to achieve D-phenothrin-degrading bacterial isolates. Isolation and enrichment of degrading bacterial isolates was carried out according to Chen et al. (2011b,c). Initial culture enrichment was performed in a $250-\mathrm{mL}$ Erlenmeyer flask containing $50 \mathrm{~mL}$ of sterilized MSM supplemented with $5 \mathrm{~g}$ of activated sludge and $50 \mathrm{mg} \cdot \mathrm{L}^{-1}$ of $\mathrm{D}$-phenothrin. Enrichment culture was incubated in an incubator shaker at $180 \mathrm{rpm}$ and $30^{\circ} \mathrm{C}$ for 7 days. After 7 days, $0.5 \mathrm{~mL}$ of enrichment culture was transferred to new Erlenmeyer flasks containing $50 \mathrm{~mL}$ of fresh MSM and 100, 200, 400, and $800 \mathrm{mg} \cdot \mathrm{L}^{-1} \mathrm{D}$-phenothrin, respectively. After five rounds of transferring, the enrichment medium was serially diluted and spread on MSM plates along with $50 \mathrm{mg} \cdot \mathrm{L}^{-1} \mathrm{D}$-phenothrin to isolate individual colonies. After purifying the obtained isolates, their D-phenothrin degradation potential was investigated. Concentrations of D-phenothrin residues in culture fluids were detected by HPLC (Waters 2690, United States).

\section{Identification and Characterization of Strain P31}

Colony and cellular morphologies of strain P31 were studied under electron microscope (BH-2 Olympus, Japan) and scanning electron microscope (XL-30 ESEM, Philips Optoelectronics Co., Ltd., Holland) on LB plates and slides, respectively.

Strain P31 was identified by sequencing $16 \mathrm{~S}$ rRNA, which is the most conserved DNA region in prokaryotes (Festa et al., 2013). Genomic DNA was prepared by using MasterPure ${ }^{\text {TM }}$ DNA Purification Kit (Epicentre Biotechnologies, United States). 16S rRNA gene was amplified with reference (Escherichia coli) primers 27F (5V-AGA GTT TGA TCC TGG CTC AG-3V) and 1492R (5V-TAC GGY TAC CTT GTT ACG ACT T-3V) and NCBI BLAST tool was used to compare 16S rRNA sequences. Bacterium was further identified by Biolog Microbial Identification System (GP2 MicroPlate ${ }^{\text {TM }}$, BIOLOG, Hayward, CA, United States). 


\section{Growth and Degradation Characteristics of Strain P31}

Strain P31 was thawed and inoculated in LB medium for $12 \mathrm{~h}$. Bacterial cells were harvested by centrifugation $(5 \mathrm{~min}, 1950 \times \mathrm{g}$ ) and washed twice with sterile saline $(0.9 \% \mathrm{NaCl})$ to prepare a concentrated inoculum solution (Chen et al., 2011a). A total of $0.3 \mathrm{~g} \cdot \mathrm{L}^{-1}$ of inoculum was transferred to $50 \mathrm{~mL}$ of MSM medium containing $50 \mathrm{mg} \cdot \mathrm{L}^{-1}$ of D-phenothrin, and kept at $29.5^{\circ} \mathrm{C}$ and $200 \mathrm{rpm}$ in a rotary shaker. Initial $\mathrm{OD}_{600}$ of the culture was about 0.05. All set-ups were prepared in triplicates, and non-inoculated cultures served as controls. Samples were collected at 0, 12, 24, $36,48,60$, and $72 \mathrm{~h}$, respectively. Residual concentrations of D-phenothrin were detected by HPLC.

\section{Optimization of D-Phenothrin Degradation Conditions}

Based on Box-Behnken design, optimized conditions of D-phenothrin biodegradation were determined according to the response surface methodology (RSM) (Ghevariya et al., 2011; Zhang et al., 2011; Chen et al., 2013). For statistical calculations, the variables $X_{\mathrm{i}}$ (the uncoded value of the $i$ th independent variable) were coded as $x_{\mathrm{i}}$ (the coded value of the $i$ th independent variable) according to the following equation:

$$
x_{i}=\left(X_{i}-X_{0}\right) / \Delta X_{i}
$$

where $x_{\mathrm{i}}$ is the value of $X_{\mathrm{i}}$ at the center point, and $\Delta X_{\mathrm{i}}$ is the step change value.

Based on the preliminary results of one-factor-at-atime experiments (data not shown), critical factors such as temperature, $\mathrm{pH}$ and inoculum size were selected as independent variables. Levels of the variables and experimental Box-Behnken design are shown in Supplementary Table 1. Accordingly, based on Box-Behnken design 15 experiments were carried out to build quadratic models, with three replications of the center points to estimate experimental error. Experimental data of the model experiments was used to predict optimal conditions according to the following equation:

$$
Y_{i}=b_{0}+\sum \mathrm{p}_{i} X_{i}+\sum \mathrm{p}_{i j} X_{i} X_{j}+\sum \mathrm{p}_{i i} X_{i i}^{2}+e_{i}
$$

where $X_{\mathrm{i}}$ are the input variables, which influence the response variable $Y_{\mathrm{i}}, \mathrm{p}_{0}$ is the offset term, $\mathrm{p}_{\mathrm{i}}, \mathrm{p}_{\mathrm{ii}}$, and $\mathrm{p}_{\mathrm{ij}}$ are the first-order, quadratic, and interaction coefficients, respectively, $i$ and $j$ are the index numbers for the factors, and $e_{i}$ is the residual error.

SAS 9.0 was used for statistical analysis and building regression model to predict optimal processing parameters.

\section{Dynamics and Substrate Range Characteristics of Strain P31}

Degradation dynamics parameters were determined by measuring specific degradation constant at various $\mathrm{D}$-phenothrin concentrations $\left(25,50,100,200,400\right.$, and $\left.800 \mathrm{mg} \cdot \mathrm{L}^{-1}\right)$ under optimal reaction conditions. D-phenothrin residual concentrations were detected and specific degradation constant (q) at different initial concentrations was assessed according to substrate inhibition model (Luong, 1987), as follows.

$$
q=\left(q_{\max } \mathrm{S}\right) / S+K_{\mathrm{s}}+\left(S^{2} / K_{\mathrm{i}}\right)
$$

where $q_{\max }$ is the maximum specific degradation constant, $K_{s}$ is the half-saturation constant, $K_{\mathrm{i}}$ is the substrate inhibition constant, and $S$ is the inhibitor concentration. Kinetic parameters were calculated by non-linear regression method in MATLAB 7.8.

To assess the degradation ability of strain P31 against various SPs, MSM medium was supplemented with D-phenothrin, permethrin, cyhalothrin, $\beta$-cypermethrin, deltamethrin, fenpropathrin, or bifenthrin at $50 \mathrm{mg} \cdot \mathrm{L}^{-1}$ along with the optimal inoculum size. Each treatment was performed in triplicate and incubated at optimum conditions whereas non-inoculated samples served as control. Samples of different treatments were collected at regular intervals and degradation was measured as described above.

\section{Identification of D-Phenothrin Metabolic Products}

To identify D-phenothrin and its metabolic products of biodegradation, the strain P31 was grown in MSM media containing $50 \mathrm{mg} \cdot \mathrm{L}^{-1}$ of D-phenothrin. Non-inoculated samples containing the same amount of D-phenothrin served as control. Samples of different treatments were collected at regular intervals as described above, and extracted after acidification to $\mathrm{pH} 2$ (2 M $\mathrm{HCl}$ ) according to Tallur et al. (2008). Metabolites were detected by gas chromatography-mass spectrometry (GC-MS) (Agilent $6890 \mathrm{~N} / 5975$, United States). To identify the metabolites, mass spectrometry analyses were matched with authentic standard compounds from the National Institute of Standards and Technology (NIST, United States) library database.

\section{Biodegradation of D-Phenothrin in Soils}

Soil samples were collected at the depth of 5-20 cm from a field, which had never been treated with D-phenothrin or fertilizers, in South China Agricultural University, Guangzhou, China. Physicochemical properties of the soils were as $(\mathrm{g} / \mathrm{kg}$ of dry weight): organic matter, 10.5 ; total $\mathrm{N}, 0.5$; total $\mathrm{P}, 0.4$; total $\mathrm{K}, 18.2$; and $\mathrm{pH}, 6.9$ and has sandy loam texture (sand, $65.0 \%$; silt, $28.0 \%$; clay, $7.0 \%$ ). Soil samples were air-dried in the laboratory to the suitable moisture level, sieved ( $2 \mathrm{~mm}$ ), and used for bioremediation studies (Chen et al., 2012, 2014).

Sterile (at $121^{\circ} \mathrm{C}$ for $1 \mathrm{~h}$ ) and nonsterile soils (nSSs) were used to investigate bioremediation ability of strain P31. A total of $600 \mathrm{~g}$ of sterile or nSSs were placed in 500-mL Erlenmeyer flasks, and D-phenothrin solution was added to a final concentration of $50 \mathrm{mg} \cdot \mathrm{kg}^{-1}$ of soil in acetone solution. Erlenmeyer flasks were kept opened to allow aerobic conditions. Water contents were adjusted to $40 \%$ of water-holding capacity with deionized water (Cycoń et al., 2014). After mixing, the strain P31 suspension was introduced into the soils by drip irrigation to a final concentration of $1.0 \times 10^{7} \mathrm{CFU} \cdot \mathrm{g}^{-1}$ of soil and incubated in a sterile incubator at $30^{\circ} \mathrm{C}$. Sterile or nSS samples having insecticides but without strain P31 suspension were used as controls. Triplicate soil 
samples were kept in a dark thermostatic chamber for 10 days at $30^{\circ} \mathrm{C}$.

Soil samples (20 g) for D-phenothrin residue analysis were collected on day $0,2,4,6,8$, and 10 . Samples were ultrasonically extracted with $40 \mathrm{~mL}$ of ethyl acetate for $15 \mathrm{~min}$, and then centrifuged at $4000 \times g$ for $6 \mathrm{~min}$ to obtain supernatants. Supernatants were pooled and loaded onto a chromatography column $(35 \mathrm{~cm}$ length $\times 1 \mathrm{~cm}$ i.d., pre-eluted with $40 \mathrm{~mL}$ of ethyl acetate), packed with anhydrous sodium sulfate, silica gel, and neutral alumina. Column was eluted three times, with $60 \mathrm{~mL}$ of ethyl acetate. Eluates were evaporated and residue was re-dissolved in $1.5 \mathrm{~mL}$ methanol for HPLC analysis.

Degradation constant $(k)$ was determined according to the first-order kinetic model (Eq. 4) (Cycoń et al., 2009):

$$
C_{t}=C_{0} \times e^{-k t}
$$

where $C_{0}$ is the initial concentration of D-phenothrin at zero time, $C_{\mathrm{t}}$ is the concentration of D-phenothrin at time $t, k$, and $t$ are the degradation constant $\left(\mathrm{day}^{-1}\right)$ and degradation period in days, respectively.

D-phenothrin half-life $\left(t_{1 / 2}\right)$ in different soils was calculated by using Eq. 5:

$$
t_{1 / 2}=\ln 2 / k
$$

where $k$ is the degradation constant $\left(\right.$ day $\left.^{-1}\right)$.

\section{Chemical Analysis}

Analysis of D-phenothrin residues in filtrates was carried on a Phenomenex $\mathrm{C}_{18}$ reversed phase column $(250 \times 4.60 \mathrm{~mm}$, $5 \mu \mathrm{m}$ ) with a Water 2690 HPLC system. A mixture of acetonitrile and water (90:10) was used as the mobile phase at the flow rate of $1.0 \mathrm{~mL} \cdot \mathrm{min}^{-1}$. Based on retention time (RT) and peak area of pure standard, array detection was carried out from 190 to $400 \mathrm{~nm}$ (total scan) at detection limit of $0.01 \mathrm{mg} \cdot \mathrm{L}^{-1}$. Detection wavelengths of D-phenothrin, permethrin, cyhalothrin, $\beta$-cypermethrin, deltamethrin, fenpropathrin, and bifenthrin were as 276.8, 276.8, 276.8, 276.8, 250.9, 276.8, and $354 \mathrm{~nm}$, respectively.

Analysis of D-phenothrin metabolic products, was performed on Agilent 6890N/5975 GC-MS system equipped with auto-sampler, an on-column, split/splitless capillary injection system with array detection from 30 to $500 \mathrm{~nm}$ (total scan). Chromatographic column was a HP-5MS capillary column $(30.0 \mathrm{~m} \times 250 \mu \mathrm{m} \times 0.25 \mu \mathrm{m})$, and column temperature was initially held at $90^{\circ} \mathrm{C}$ for $2 \mathrm{~min}$, then increased to $150^{\circ} \mathrm{C}$ at the rate of $6^{\circ} \mathrm{C} \cdot \mathrm{min}^{-1}$ for $1 \mathrm{~min}$, raised to $180^{\circ} \mathrm{C}$ at the rate of $10^{\circ} \mathrm{C} \cdot \mathrm{min}^{-1}$ for $4 \mathrm{~min}$ and finally increased to $260^{\circ} \mathrm{C}$ at the rate of $20^{\circ} \mathrm{C} \cdot \mathrm{min}^{-1}$ for $10 \mathrm{~min}$. Carrier gas (helium) flow rate was $1.5 \mathrm{~mL} \cdot \mathrm{min}^{-1}$ and the injection volume was $1.0 \mu \mathrm{L}$ with splitless sampling at $250^{\circ} \mathrm{C}$. Temperatures corresponding to transfer line and the ion trap were $280^{\circ} \mathrm{C}$ and $230^{\circ} \mathrm{C}$, respectively, and the ionization energy was $70 \mathrm{eV}$ (Chen et al., 2014, 2015).

\section{Nucleotide Sequence Accession Number}

The GenBank accession number of strain P31 16S rRNA gene is KY229868.

\section{RESULTS}

\section{Isolation of D-Phenothrin-Degrading Strain}

Based on the isolation and enrichment results, 34 morphologically different strains (grown on MSM containing $800 \mathrm{mg} \cdot \mathrm{L}^{-1} \mathrm{D}$-phenothrin) were attained from activated sludge and named as P01 34, respectively. Results showed that strain P31 possessed the highest degradation capacity among 34 strains and degraded $100 \%$ of D-phenothrin within $72 \mathrm{~h}$ of incubation. Degradation of D-phenothrin by each isolated strain within $72 \mathrm{~h}$ of incubation is shown in Supplementary Table 2. Moreover, strain P31 was also found to efficiently utilize permethrin, cyhalothrin, $\beta$-cypermethrin, deltamethrin, fenpropathrin, and bifenthrin as the sole source of carbon. Thus, strain P31 was selected for further studies.

\section{Identification and Characterization of Strain P31}

Physio-biochemical properties of the strain P31 are shown in Supplementary Table 3. Strain P31 is a Gram-negative, obligatory aerobic and rod-shaped bacterium of $1.5 \sim 4.0 \mu \mathrm{m}$ length and $0.5 \sim 1.0 \mu \mathrm{m}$ width. Colonies grown on LB agar plates were primrose yellow, opaque, and circular with smooth margin. This bacterial isolate showed positive reactions in catalase, oxidase, citrate utilization, nitrate reduction, hemolysis, and arginine dihydrolase tests, but remained negative in anaerobic growth, gelatin liquefaction, urease production, indole production, and esculin hydrolysis tests. Growth was observed over a temperature range of $20 \sim 40^{\circ} \mathrm{C}$, a salinity range of $2 \sim 5 \% \mathrm{NaCl}$, and a $\mathrm{pH}$ range of $5 \sim 10$. It was positive in the utilization of glycerol, $\alpha$-D-glucose, $\mathrm{D}$-fructose, L-fucose, L-alanine, dextrin, L-serine, inosine, $\mathrm{D}$-galactose, $\mathrm{D}$-fucose, methyl pyruvate, sodium bromate; and negative in the utilization of sucrose, pectin, gelatin, D-maltose, D-trehalose, D-cellobiose, stachyose, Dturanose, gentiobiose, D-raffinose, D-melibiose, and $\beta$-methyl-Dglucoside.

According to BLAST analysis, partial sequence showed high similarity to $16 \mathrm{~S}$ rRNA gene sequence of Pseudomonas group and closely clustered with strain f2 (2012) (GenBank accession No. JQ717288) and strain SRZ19 (GenBank accession No. KU877341), having sequence identities over 99\%. Similarity and distance between strain P31 and the model strain ( $P$. fulva) were 0.617 and 4.355 , respectively, according to Biolog microbial identification system. Therefore, the strain P31 was confirmed as P. fulva based on the morphology, physio-biochemical properties, $16 \mathrm{~S}$ rRNA gene analysis, and Biolog microbial identification system.

\section{Growth and Degradation Characteristics of Strain P31}

Growth of strain P31 in MSM media with D-phenothrin as the sole carbon resource and its degradation characteristics are shown in Figure 1. Strain P31 showed rapid growth without lag phase during initial period $(0 \sim 12 \mathrm{~h})$ indicating that the strain utilized D-phenothrin as growth substance. 


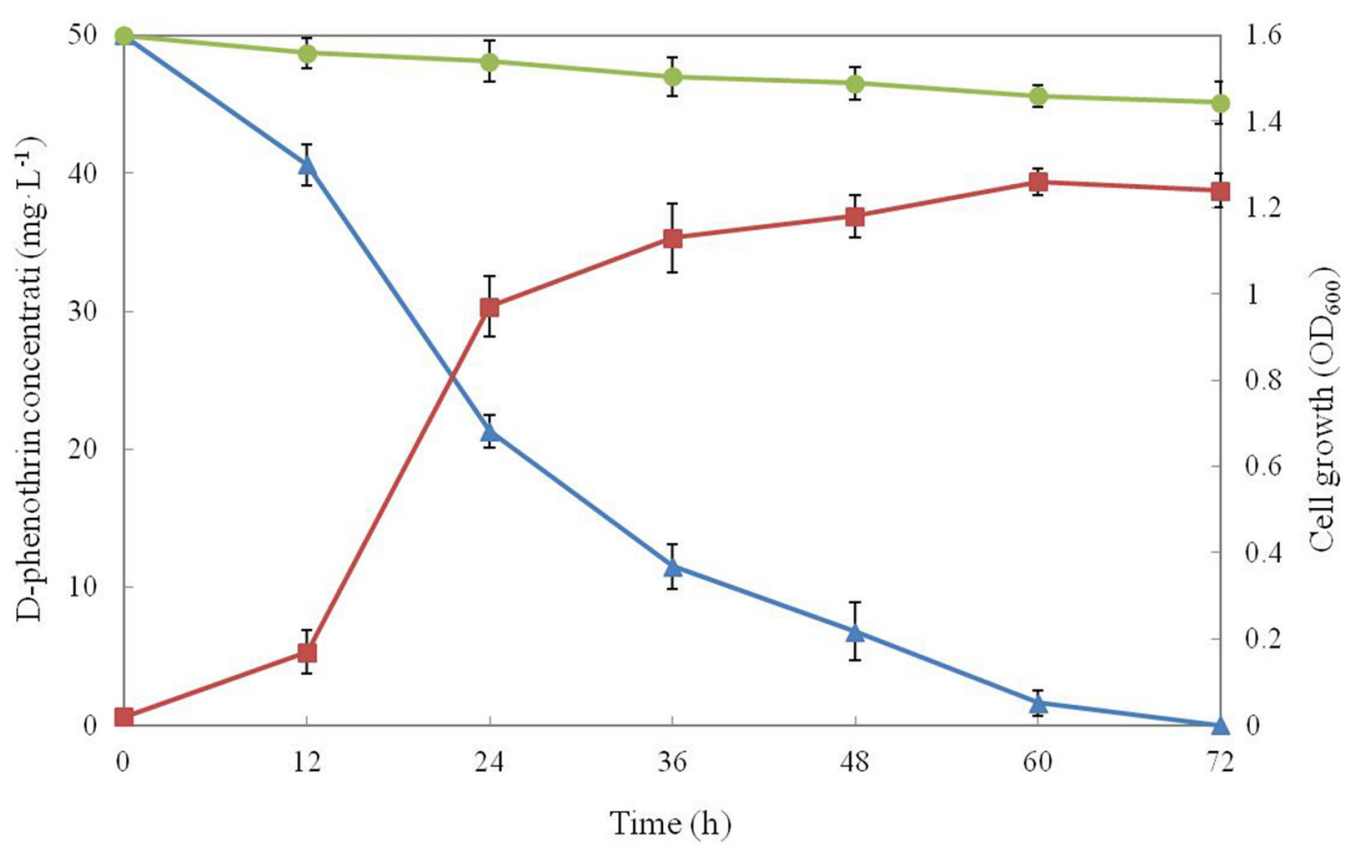

FIGURE 1 | Biodegradation of D-phenothrin $\left(50 \mathrm{mg} \cdot \mathrm{L}^{-1}\right)$ during growth of strain P31. Symbol: $\bullet$, D-phenothrin control; $\mathbf{\Lambda}$, D-phenothrin degradation by strain P31; 口, cell growth. Data represent mean values of three replicates with standard deviation.

Cell growth hiked after $12 \mathrm{~h}$ of incubation, and significant degradation of D-phenothrin was noted during this period. Cell growth increased rapidly at logarithmic phase (12 24 h), and approximately $57.0 \%$ of the D-phenothrin was degraded by strain P31 in $24 \mathrm{~h}$. During this time, the concentration of D-phenothrin decreased with a concomitant increase of cell number. Subsequently, cell growth slowed down at stationary phase $(36 \sim 60 \mathrm{~h})$, and about $77.0-86.4 \%$ of the initial dose was eliminated within 36-48 h, respectively. Strain P31 reached to maximum level of growth at $60 \mathrm{~h}$ post inoculation and caused the maximum D-phenothrin biodegradation of $96.8 \%$. Finally, the strain did not show growth at decline phase $(>72 \mathrm{~h})$ and the residual amount of D-phenothrin was not detectable by HPLC at $72 \mathrm{~h}$ post incubation. Approximately $9.0 \%$ degradation was observed in the non-inoculated control.

\section{Optimization of D-Phenothrin Degradation Conditions}

Supplementary Table 1 demonstrates the level and range of three independent variables, including temperature $\left(X_{1}\right), \mathrm{pH}\left(X_{2}\right)$, and inoculum size $\left(X_{3}\right)$. The three parameters were taken at a central coded value considered as zero and investigated at three different levels $(-1,0$, and +1$)$. In this case, the result of a three-level factorial design, a total number of 15 experiments were employed to match the second-order polynomial model in accordance with Box-Behnken design. Statistical combinations of critical parameters along with the maximum observed and predicted degradation are displayed in Supplementary Table 1. These predicted values were in close proximity to the observed ones in all sets of experiments. Highest degradation of $100 \%$ and the lowest of $80.4 \%$ were observed. Two regression equations, Eq. 6 a for coded values and Eq. 6 b for actual experimental values, which are analogous to Eq. 2 , showed the degradation rate $(Y)$ as a function of the test variables temperature $\left(X_{1}\right), \mathrm{pH}\left(X_{2}\right)$, and inoculum size $\left(X_{3}\right)$.

$$
\begin{aligned}
Y= & 100-1.9375 X_{1}+1.35 X_{2}+2.7375 X_{3}-9.4875 X_{1}^{2} \\
& +0.2 X_{1} X_{2}-0.075 X_{1} X_{3}-5.5625 X_{2}^{2}+1.45 X_{2} X_{3} \\
& -1.1375 X_{3}^{2} \\
Y= & -494.163+22.1325 X_{1}+75.125 X_{2}-24.125 X_{3} \\
& -0.3795 X_{1}^{2}+0.04 X_{1} X_{2}-0.15 X_{1} X_{3}-5.5625 X_{2}^{2} \\
& +14.5 X_{2} X_{3}-113.75 X_{3}^{2}
\end{aligned}
$$

Analysis of variance (ANOVA) is essential to test the significance and adequacy of the model (Ghevariya et al., 2011) and ANOVA of the quadratic regression model is tabulated in Supplementary Table 4. As shown in the table, a very low probability value $(P$-value $<0.05)$, a large value of the regression coefficient $\left(R^{2}=0.9905\right)$ and a low coefficient of variation $(\mathrm{CV}=1.10)$ demonstrated that the established quadratic polynomial model for D-phenothrin degradation by strain P31 presented the actual relationship of response and variables. Regression analysis reveals that the linear terms of temperature $\left(X_{1}\right), \mathrm{pH}\left(X_{2}\right)$, and inoculum size $\left(X_{3}\right)$ are important factors in the D-phenothrin degradation by strain P31. Square terms of $X_{1}^{2}$ 
and $\mathrm{pH} X_{2}^{2}$, interaction terms of $X_{1} X_{3}$ and $X_{2} X_{3}$ also showed significant effects $(P<0.05)$ on $\mathrm{D}$-phenothrin degradation by strain P31, however, square terms of $X_{3}^{2}$ and interaction term of $X_{1} X_{2}$ played insignificant role $(P>0.05)$ in degradation (Supplementary Table 4).

For better understanding of the results, a 3D response surface plot is given in Supplementary Figure 2, which illustrates the effects of temperature $\left(X_{1}\right)$ and $\mathrm{pH}\left(X_{2}\right)$ on D-phenothrin degradation with inoculum size $\left(X_{3}\right)$ as a constant. Supplementary Figure 2 has clear stationary point, indicating that maximum degradation could be achieved inside the design boundaries. Model depicted maximum degradation of $100 \%$ at temperature $29.5^{\circ} \mathrm{C}, \mathrm{pH} 7.3$ and an inoculum size of $0.3 \mathrm{~g} \cdot \mathrm{L}^{-1}$. Thus, the optimum culturing conditions for D-phenothrin degradation by strain P31 were concluded to be temperature $29.5^{\circ} \mathrm{C}, \mathrm{pH} 7.3$ and an inoculum size of $0.3 \mathrm{~g} \cdot \mathrm{L}^{-1}$.

\section{Biodegradation Kinetics of D-Phenothrin by Strain P31}

$\mathrm{D}$-phenothrin biodegradation at different initial D-phenothrin concentrations is presented in Figure 2A. Strain P31 utilized and degraded D-phenothrin up to a concentration of $800 \mathrm{mg} \cdot \mathrm{L}^{-1}$, and no lag period was observed. D-phenothrin was degraded completely within $72 \mathrm{~h}$ at initial concentrations below $50 \mathrm{mg} \cdot \mathrm{L}^{-1}$. During $72 \mathrm{~h}$ incubation, strain P31 displayed $91.2,88.3,82.6$, and $80.4 \%$ of degradation at D-phenothrin initial concentrations of $100,200,400$, and $800 \mathrm{mg} \cdot \mathrm{L}^{-1}$, respectively. Increased concentration of $\mathrm{D}$-phenothrin had a slight effect on biodegradation performance of strain P31, indicating that the $\mathrm{D}$-phenothrin degradation by this strain is concentration dependent. Relationship between initial D-phenothrin concentration and specific degradation constant by strain P31 is shown in Figure 2B. According to the substrate inhibition model (Eq. 3), $K_{s}$ and $K_{\mathrm{i}}$ values were determined by non-linear regression method as 3.5066 and $482.1673 \mathrm{mg} \cdot \mathrm{L}^{-1}$, respectively. Critical inhibitor concentration $\left(S_{m}\right)$ was calculated as $41.1189 \mathrm{mg} \cdot \mathrm{L}^{-1}$ whereas the overall $q_{\max }$ value remained $0.0455 \mathrm{~h}^{-1}$. As shown in Figure 2B, at the initial concentration of $\mathrm{D}$-phenothrin below $50 \mathrm{mg} \cdot \mathrm{L}^{-1}$, $q$ value was rapidly increased. At the initial concentrations of D-phenothrin above $100 \mathrm{mg} \cdot \mathrm{L}^{-1}, q$ value gradually declined with the increase of $\mathrm{D}$-phenothrin. These results illustrated that the biodegradation of $\mathrm{D}$-phenothrin by strain

A

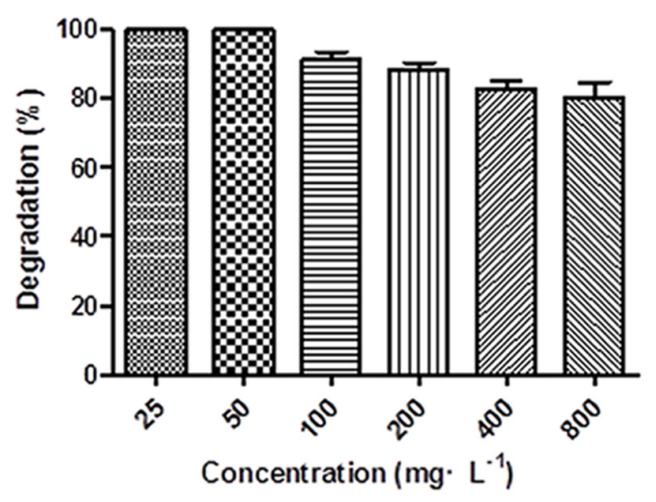

B

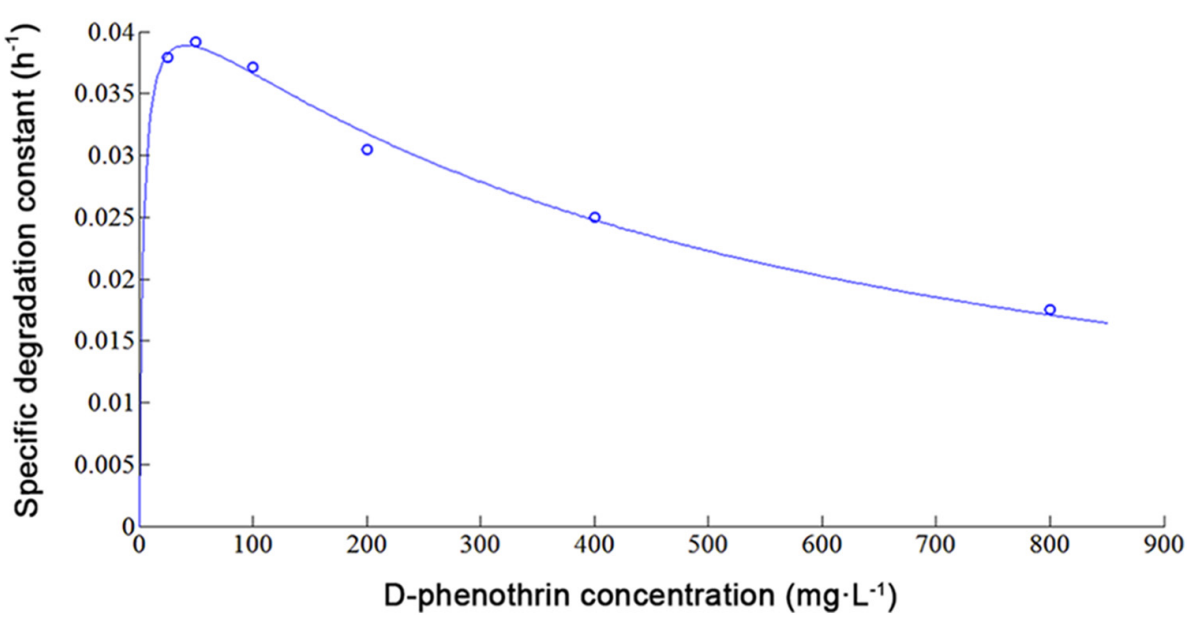

FIGURE 2 | Biodegradation of D-phenothrin at different initial concentrations by strain P31 within $72 \mathrm{~h}$. (A) Degradation of D-phenothrin at different concentrations; (B) Relationship between initial D-phenothrin concentration and specific degradation constant by strain P31. Data represent mean values of three replicates with standard deviation. 
P31 could be partially inhibited at high concentration of D-phenothrin.

\section{Biodegradation Kinetics of Various SPs}

Strain P31 revealed capability to utilize various SPs as food source including D-phenothrin, permethrin, cyhalothrin, $\beta$-cypermethrin, deltamethrin, fenpropathrin, and bifenthrin, having degradation efficiency of 100, 96.7, 89.2, 86.6, 83.1, 71.4, and $57.9 \%$ within $72 \mathrm{~h}$, respectively (Figure 3 ). To further quantify the biodegradation capacity of strain P31 against different SPs, the degradation constant $(k)$ and half-life $\left(t_{1 / 2}\right)$ were determined by using the first-order kinetic model. Degradation kinetic parameters of various SPs by strain P31 are summarized in Table 1. Biodegradation process was characterized by a $k$ ranging from 0.0117 to $0.0392 \mathrm{~h}^{-1}$. The $t_{1 / 2}$ value of the above mentioned SPs with strain P31 were calculated as $17.7,21.0,23.7,25.9,27.4,36.9$, and $59.2 \mathrm{~h}$, respectively, and are significantly lower as compared to those in the environment.

\section{Metabolic Products and Degradation Pathway of D-Phenothrin}

Samples obtained at different intervals were analyzed through GC-MS to identify the metabolic products during the bacterial

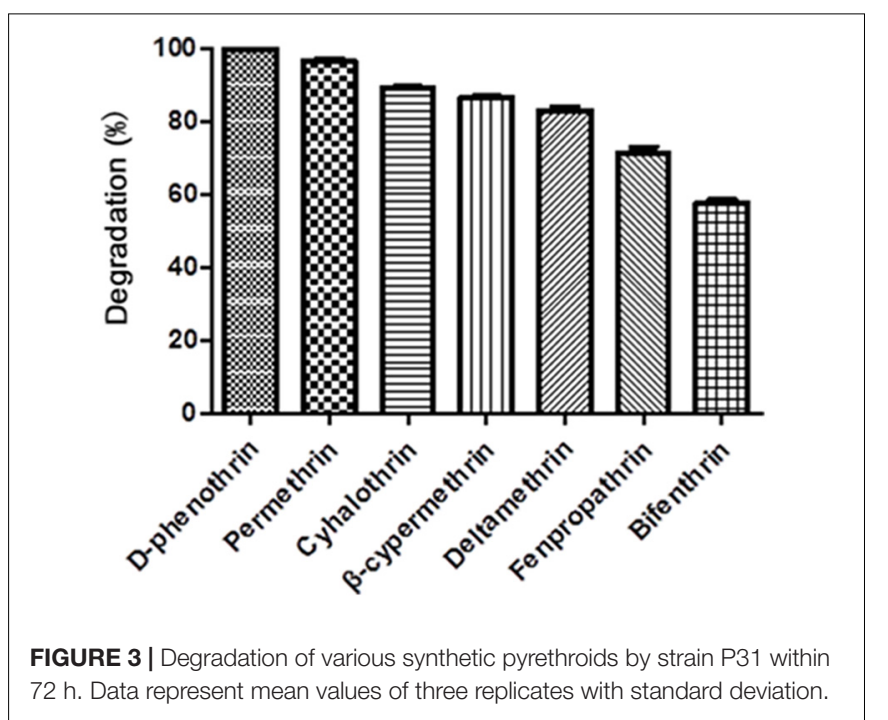

TABLE 1 | Kinetic parameters of degradation of synthetic pyrethroids by strain P31.

\begin{tabular}{lllll}
\hline $\begin{array}{l}\text { Synthetic } \\
\text { pyrethroids }\end{array}$ & Regression equation & $\left.\boldsymbol{k} \mathbf{( h}^{-\mathbf{1}}\right)$ & $\boldsymbol{t}_{\mathbf{1 / 2}} \mathbf{( h )}$ & $\boldsymbol{R}^{\mathbf{2}}$ \\
\hline D-phenothrin & $C_{\mathrm{t}}=53.5150 \mathrm{e}^{-0.0392 t}$ & 0.0392 & 17.7 & 0.9802 \\
Permethrin & $C_{\mathrm{t}}=53.8361 \mathrm{e}^{-0.0330 t}$ & 0.0330 & 21.0 & 0.9609 \\
Cyhalothrin & $C_{\mathrm{t}}=53.1980 \mathrm{e}^{-0.0292 t}$ & 0.0292 & 23.7 & 0.9848 \\
$\beta$-cypermethrin & $C_{\mathrm{t}}=53.3589 \mathrm{e}^{-0.0268 t}$ & 0.0268 & 25.9 & 0.9822 \\
Deltamethrin & $C_{\mathrm{t}}=52.1891 \mathrm{e}^{-0.0253 t}$ & 0.0253 & 27.4 & 0.9736 \\
Fenpropathrin & $C_{\mathrm{t}}=57.7978 \mathrm{e}^{-0.0188 t}$ & 0.0188 & 36.9 & 0.9902 \\
Bifenthrin & $C_{\mathrm{t}}=53.7381 \mathrm{e}^{-0.0117 t}$ & 0.0117 & 59.2 & 0.9687
\end{tabular}

degradation process. In the initial sample of 0 and $12 \mathrm{~h}$, a significant peak was detected at RT of $26.710 \mathrm{~min}$, displaying a characteristic mass fragment $\left[\mathrm{M}^{+}\right]$at $\mathrm{m} / \mathrm{z} 350$ with major fragment ions at $m / z 123$ and 183, which was identified as D-phenothrin and named as compound A. Subsequently, the compound A disappeared concomitantly with the formation of two new compounds. According to the similarity of their fragment RTs and molecular ions to those of corresponding authentic compounds in the NIST library database, the two compounds were characterized as 3-phenoxybenzaldehyde (compound B) and 1,2-benzenedicarboxylic butyl dacyl ester (compound C), respectively (Supplementary Figure 3). It is the first time that these metabolic products were detected in the degradation pathway of D-phenothrin. It is noteworthy that all these metabolites were transitory and faded away without any non-cleavable metabolites at the end of experiment. Chemical structures, RT, and characteristic ions of the mass spectra are shown in Figure 4.

A degradation pathway of D-phenothrin in strain P31 was proposed based on the chemical structures of D-phenothrin and metabolites formed during fermentation (Figure 5). D-phenothrin was first hydrolyzed by cleavage of the carboxylester linkage to produce 3-phenoxybenzaldehyde and $\quad(1 R, 3 R)$-trans-2,2-dimethyl-3-(2-methyl-1-propenyl) cyclopropane-1-carboxylic acid. Then, the intermediate product 3-phenoxybenzaldehyde was further degraded with diaryl cleavage to form 1,2-benzenedicarboxylic butyl dacyl ester. Eventually, D-phenothrin was degraded by strain P31 without any persistent accumulative product.

\section{Biodegradation of D-Phenothrin in Soils}

The ability of strain P31 to degrade D-phenothrin in contaminated soils was evaluated under controlled conditions (Figure 6). Degradation process was further investigated using the first-order kinetic model as shown in Table 2. D-phenothrin degraded rapidly after strain P31 inoculation $\left(1 \times 10^{7} \mathrm{CFU} \cdot \mathrm{g}^{-1}\right.$ of soil) in nSS at the beginning of incubation, and approximately $77.0 \%$ of D-phenothrin at initial concentration of $50 \mathrm{mg} \cdot \mathrm{kg}^{-1}$ was removed within 10 days without any lag phase. Degradation process was characterized by a degradation constant $(k)$ of 0.1380 day $^{-1}$ and $t_{1 / 2}$ value of 5.0 days (Table 2 ). By contrast, only $34.6 \%$ of added pesticide was degraded in the control group inoculated with autochthonous microorganisms, at $t_{1 / 2}$ value of 18.5 days. About $75.4 \%$ of initial D-phenothrin was removed from the sterile soil (SS) within 10 days at $k$-value of 0.1167 day $^{-1}$ and $t_{1 / 2}$ of 5.9 days. In the non-inoculated control, this activity decreased to only $19.1 \%$ in SS at $t_{1 / 2}$ of 32.7 days. It is noteworthy that the degradation of D-phenothrin in nSS was higher as compared to SS.

\section{DISCUSSION}

Pseudomonas bacterial strains are known for their metabolic capability, environmental versatility and ability to degrade different xenobiotic compounds including permethrin (Maloney et al., 1988), 3-phenoxybenzoate (Topp and Akhtar, 1991), 
A Abundance

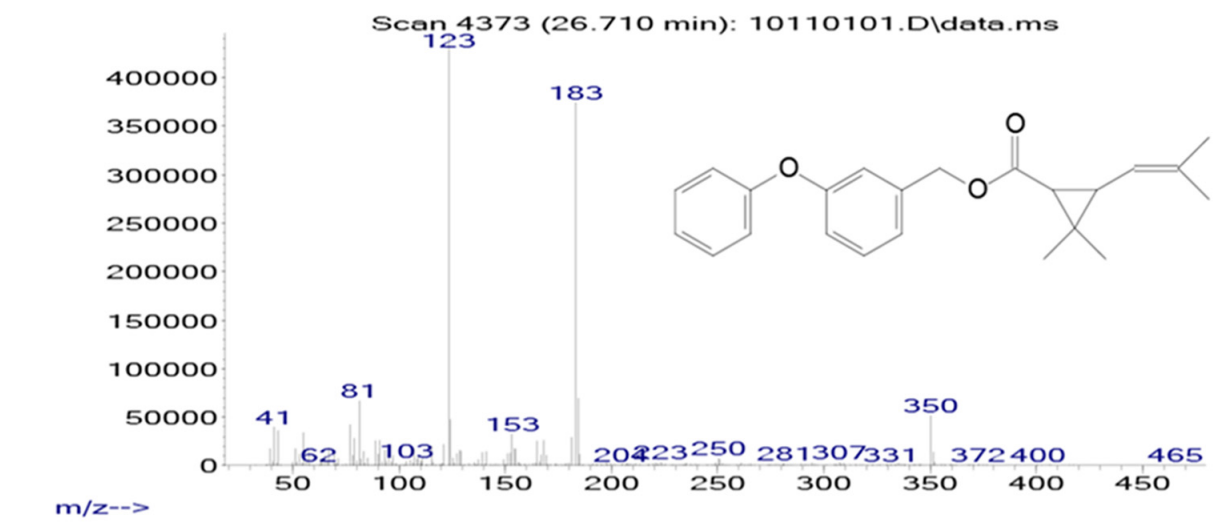

B

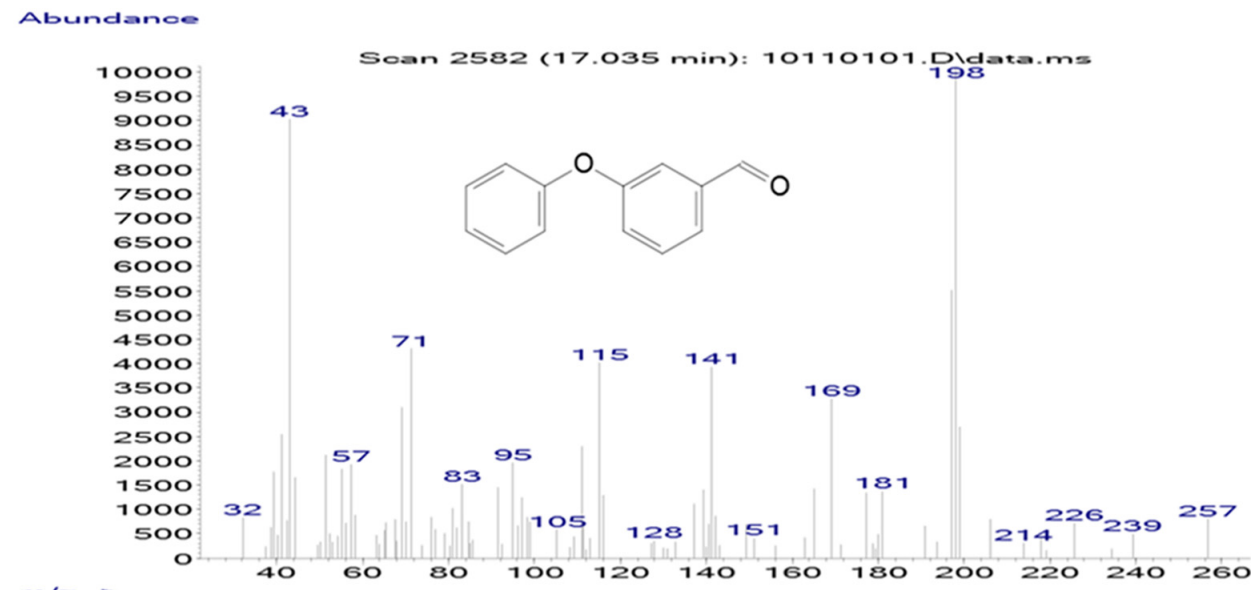

C

Abundance

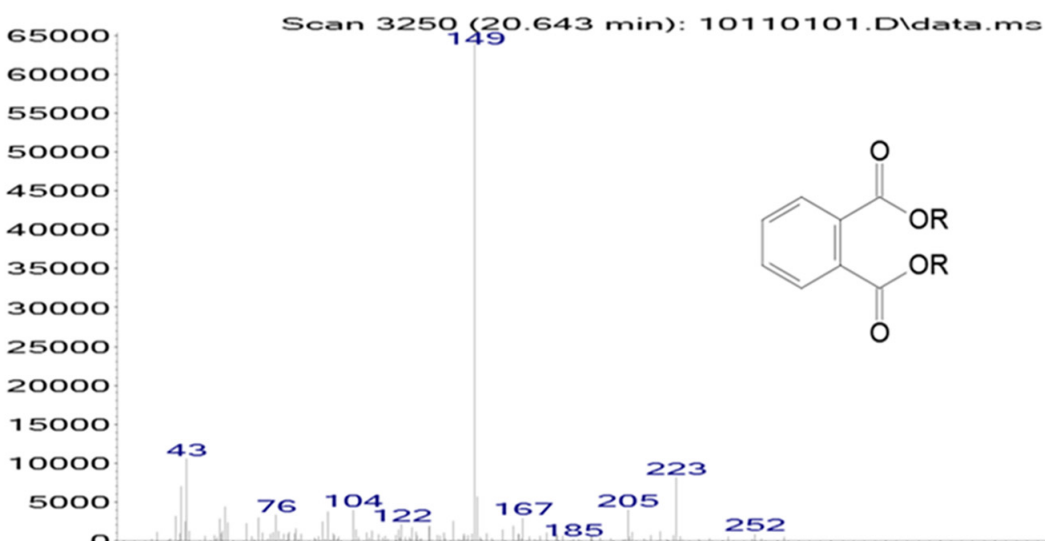

785,252 $m / z-->$

FIGURE 4 | GC-MS analysis of the metabolites produced from D-phenothrin degradation by strain P31. (A-C) The characteristic ions of compounds A-C in GC-MS. Retention time of each compound was 26.710, 17.035, and $20.643 \mathrm{~min}$, respectively, and identified as D-phenothrin, 3-phenoxybenzaldehyde, and 1,2-benzenedicarboxylic butyl dacyl ester, respectively. 


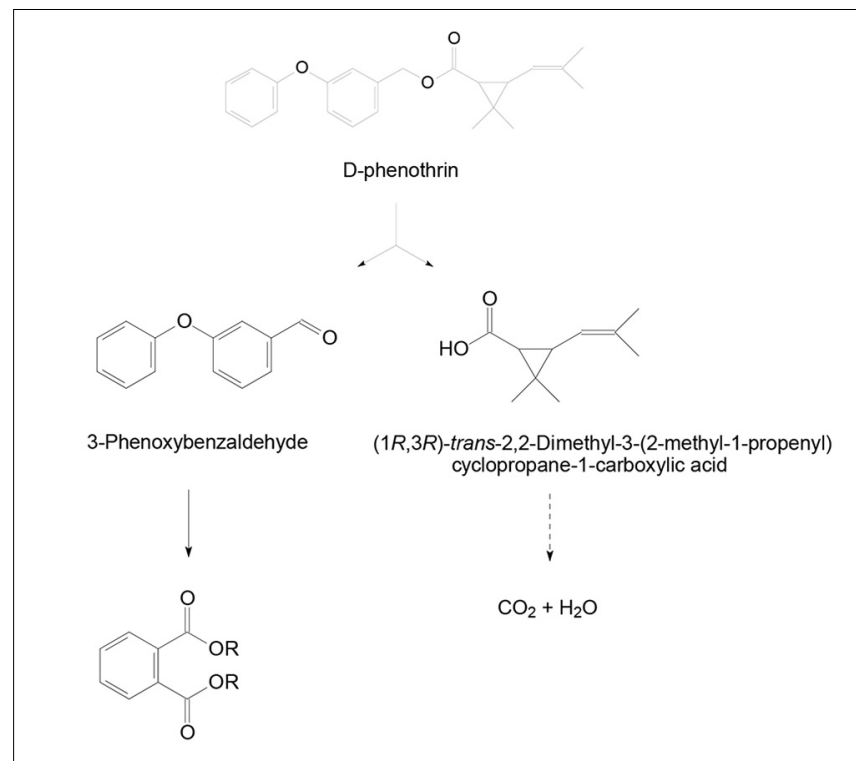

1,2-Benzenedicarboxylic butyl dacyl ester

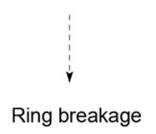

FIGURE 5 | Proposed degradation pathway of D-phenothrin in strain P31.

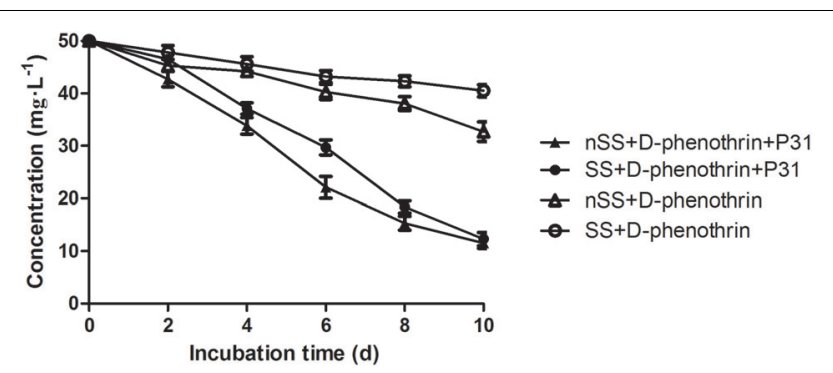

FIGURE 6 | Degradation kinetics of D-phenothrin in sterile and nSSs. Data represent mean values of three replicates with standard deviation. SS refers to sterile soils; nSS refers to nonsterile soils.

$\beta$-cyfluthrin (Saikia et al., 2005), cypermethrin (Jilani and Khan, 2006), diazinon (Cycoń et al., 2009), 4-chloro-2-nitrophenol (Arora and Bae, 2014), 4-chloro-3-nitrophenol (Arora et al., 2014), fenpropathrin (Song et al., 2015), and $\beta$-cypermethrin (Zhang et al., 2011; Tang et al., 2017). Pseudomonas fulva, one of the highly potent degrading microbes of genus Pseudomonas, is a ubiquitous environmental bacterium. Nevertheless, the potential use of P. fulva in biodegradation of organic compounds has not received the attention it deserves. Our studies demonstrated that $P$. fulva can utilize various organic pollutants as food source and thus it can be successfully colonized in nutrient deficient niches.

Generally, it is considered that the screening and enrichment of environmental microorganisms is of great importance for selecting desirable degrading bacteria (Yang et al., 2011; Liu et al., 2015; Xiao et al., 2015). In this study, 34 morphologically different strains, which grew well in MSM with D-phenothrin as the sole carbon source, were selected from pyrethroid-contaminated active sludge through enrichment culture technique, and the most active bacterium strain P31 was chosen for further studies. This is the first time that a D-phenothrin-degrading strain P31 was isolated which completely degraded D-phenothrin in $72 \mathrm{~h}$. Unlike other environmental bacteria, $P$. fulva $\mathrm{P} 31$ has remarkable capacity to degrade a variety of SPs including D-phenothrin, permethrin, cyhalothrin, $\beta$-cypermethrin, deltamethrin, fenpropathrin, and bifenthrin which are widely used insecticides and are associated with environmental contamination. Study reveals that this isolate has great potential as a degrader in cleaning-up pyrethroid residues from the environment (Delgado-Moreno et al., 2011; Kuivila et al., 2012; Markle et al., 2014).

The RSM provides reliable information for the optimization of key process parameters to enhance biodegradation by employing polynomial equation of empirical model to experimental data (Bezerra et al., 2008). Box-Behnken design has great advantages as it requires fewer experimental runs to efficiently estimate quadratic surfaces for the optimization of responses (Ghevariya et al., 2011). During this study, RSM based on Box-Behnken design was successfully explored to improve the biodegradation process in strain P31, and optimum D-phenothrin biodegradation conditions were determined as $29.5^{\circ} \mathrm{C}, \mathrm{pH} 7.3$, and an inoculum size of $0.3 \mathrm{~g} \cdot \mathrm{L}^{-1}$. In addition, two mathematical models (Eqs. 6a and 6b) could be used to predict and optimize D-phenothrin degradation conditions by strain P31 within given parameters. Models indicated maximum degradation rate of $100 \%$ under the optimum conditions. Similar enhanced degradation through RSM has been reported in a variety of degrading microbes such as Achromobacter xylosoxidans CG542 (Ghevariya et al., 2011), Pseudomonas aeruginosa $\mathrm{CH} 7$ (Zhang et al., 2011), Brevibacterium aureum DG-12 (Chen et al., 2013), and Bacillus subtilis BSF01 (Xiao et al., 2015).

TABLE 2 | Kinetic parameters of degradation of D-phenothrin in sterile and nonsterile soils.

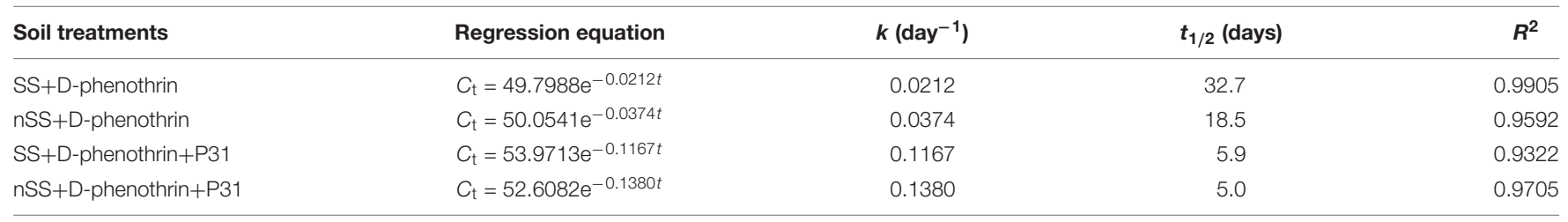

SS refers to sterile soils; $n S S$ refers to nonsterile soils. 
Biodegradation process is often inhibited by high concentration of substrates, which limits their use in bioremediation of variable environments. Previous studies reported that the metabolic activity of pyrethroid-degrading microbes lead to complete catabolite repression at high substrate concentrations $<200 \mathrm{mg} \cdot \mathrm{L}^{-1}$ (Jilani and Khan, 2006; Lin et al., 2011). Contrarily, increased concentrations of D-phenothrin did not cause much effect on the biodegradation performance of strain P31, and more importantly it did not inhibit cell growth (Figure 2B). It is noteworthy that the isolate tolerated and rapidly degraded D-phenothrin up to a concentration, as high as $800 \mathrm{mg} \cdot \mathrm{L}^{-1}$, at $K_{\mathrm{i}}$ and $q_{\max }$ of $482.1673 \mathrm{mg} \cdot \mathrm{L}^{-1}$ and $0.0455 \mathrm{~h}^{-1}$, respectively. This feature provides significant advantage to survive under various environments and utilize even the high concentrations of xenobiotic (Chen et al., 2013, 2014).

Hydrolysis plays a significant role in the biodegradation of SPs, which could be attributed to the presence of cyclopropane carboxylic acid moieties connected to aromatic alcohols with a central ester (or ether) bond in SPs. Ester bond is generally considered susceptible to degrading microbes (Stok et al., 2004; Heidari et al., 2005; Tallur et al., 2008; Wang et al., 2009; Zhang et al., 2011; Chen et al., 2015; Tang et al., 2017). In this study, D-phenothrin was initially hydrolyzed via cleavage of the ester bond to yield the main intermediate products 3-phenoxybenzaldehyde and 1,2-benzenedicarboxylic butyl dacyl ester (Figure 4). The 3-Phenoxybenzaldehyde is a common product of type II SPs, as also was detected in the biodegradation process of fenvalerate (Chen et al., 2011b), deltamethrin (Chen et al., 2011c), fenpropathrin (Chen et al., 2014), and $\beta$-cypermethrin (Xiao et al., 2015; Tang et al., 2017). However, this metabolite has been rarely observed in type I SPs. Involvement of metabolite 1,2benzenedicarboxylic butyl dacyl ester in SPs biodegradation has never been reported. It is worth noting that the intermediate products were transient and disappeared gradually, without further accumulation of metabolites at the end of experiment, suggesting that the isolate may harbor a complete metabolic pathway for the degradation and metabolism of D-phenothrin. Based on metabolites analysis, a degradation pathway of D-phenothrin in strain P31 has been proposed during this study (Figure 5). The proposed D-phenothrin degradation pathway may provide new insights into the biogeocycle of this pesticide.

During this study, potential of the strain P31 for bioremediation of pyrethroid-contaminated soils was investigated under laboratory conditions. Bioaugmentation of D-phenothrin-contaminated soils with strain P31 significantly enhanced the degradation of D-phenothrin, and 75.4 77.0\% of the initial dose of D-phenothrin $\left(50 \mathrm{mg} \cdot \mathrm{kg}^{-1}\right)$ was removed from soils within 10 days at $t_{1 / 2}$ value of 5.0 5.9 days; while in the control group without strain P31 it took 18.5 32.7 days. Results indicate biodegradation as the primary mechanism of SPs dissipation in the environment whereas its abiotic breakdown is less important (Khan et al., 1988; Maloney et al., 1988). Notably, at a final bacterial count of $1 \times 10^{7}$ cells. $\mathrm{g}^{-1}$ of soil, strain P31 rapidly degraded D-phenothrin in the beginning of incubation without any lag phase. These results contradict the findings of Cycon et al. (2014) who reported the slow degradation of deltamethrin during initial 14 days, in the soil bioaugmented with Serratia marcescens DeI- 1 and DEI-2 and only $10 \sim 14 \%$ of the initial dose was removed. Usually, the introduced bacteria need to adapt to the soil conditions, xenobiotics and to the proliferation of autochthonous microorganisms utilizing this pollutant as a carbon and energy source (Zhang et al., 2012; Cycoń and Piotrowska-Seget, 2016). Unlike other bacterial isolates, $P$. fulva P31 has an exceptional ability to adapt and thrive in nutrient deficient ecological niches that makes it a potent strain for various applications.

\section{CONCLUSION}

D-phenothrin-degrading bacterial strain P. fulva P31 was isolated and characterized in terms of its physiology, biochemistry, and biodegradation ability. Strain P31 revealed remarkable capacity to degrade a wide range of SPs that cause environmental contaminations. Based on metabolites analysis, we also proposed a D-phenothrin degradation pathway in strain P31. Moreover, this particular strain exhibited great advantages in bioremediation of pyrethroid-contaminated soils because of its adaptability in water and soil environments. These insights will facilitate to develop new strategies to curb pesticide residues related environmental pollution.

\section{AUTHOR CONTRIBUTIONS}

SC designed the experiments; JY, YF, HZ, KZ, and SC performed the experiments; JY, FY, and SC performed the data analysis; LZ provided the scientific expertise; JY, JL, FY, and SC wrote the manuscript.

\section{FUNDING}

This study was partially funded by grants from the National Natural Science Foundation of China (31401763), the National Key Project for Basic Research (2015CB150600), Guangdong Natural Science Funds for Distinguished Young Scholar (2015A030306038), the Science and Technology Planning Project of Guangdong Province (2016A020210106, 2017A010105008), and Pearl River S\&T Nova Program of Guangzhou (201506010006).

\section{SUPPLEMENTARY MATERIAL}

The Supplementary Material for this article can be found online at: https://www.frontiersin.org/articles/10.3389/fmicb. 2018.01003/full\#supplementary-material 


\section{REFERENCES}

Ansari, A. R., Rahman, S., Kaur, M., Anjum, S., and Raisuddin, S. (2011). In vivo cytogenetic and oxidative stress-inducing effects of cypermethrin in freshwater fish, Channa punctata Bloch. Ecotoxol. Environ. Saf. 74, 150-156. doi: 10.1016/ j.ecoenv.2010.08.036

Arora, P. K., and Bae, H. (2014). Biotransformation and chemotaxis of 4-chloro-2nitrophenol by Pseudomonas sp. JHN. Microb. Cell Fact. 13:110. doi: 10.1186/ s12934-014-0110-7

Arora, P. K., Srivastava, A., Garg, S. K., and Singh, V. P. (2017). Recent advances in degradation of chloronitrophenols. Bioresour. Technol. 250, 902-909. doi: 10.1016/j.biortech.2017.12.007

Arora, P. K., Srivastava, A., and Singh, V. P. (2014). Degradation of 4-chloro-3nitrophenol via a novel intermediate, 4-chlororesorcinol by Pseudomonas sp. JHN. Sci. Rep. 4:4475. doi: 10.1038/srep04475

Atmaca, E., and Aksoy, A. (2015). D-Phenothrin-induced oxidative DNA damage in rat liver and kidney determined by HPLC-ECD/DAD. Environ. Toxicol. 30, 607-613. doi: 10.1002/tox.21938

Bezerra, M. A., Santelli, R. E., Oliveir, E. P., Villar, L. S., and Escaleira, L. A. (2008). Response surface methodology (RSM) as a tool for optimization in analytical chemistry. Talanta 76, 965-977. doi: 10.1016/j.talanta.2008.05.019

Brander, S. M., Gabler, M. K., Fowler, N. L., Connon, R. E., and Schlenk, D. (2016). Pyrethroid pesticides as endocrine disruptors: molecular mechanisms in vertebrates with a focus on fishes. Environ. Sci. Technol. 50, 8977-8992. doi: 10.1021/acs.est.6b02253

Chen, S. H., Chang, C. Q., Deng, Y. Y., An, S. W., Dong, Y. H., Zhou, J. N., et al. (2014). Fenpropathrin biodegradation pathway in Bacillus sp DG-02 and its potential for bioremediation of pyrethroid-contaminated soils. J. Agric. Food Chem. 62, 2147-2157. doi: 10.1021/jf404908j

Chen, S. H., Deng, Y. Y., Chang, C. Q., Lee, J., Cheng, Y. Y., Cui, Z. N., et al. (2015). Pathway and kinetics of cyhalothrin biodegradation by Bacillus thuringiensis strain ZS-19. Sci. Rep. 5:8784. doi: 10.1038/srep08784

Chen, S. H., Dong, Y. H., Chang, C. Q., Deng, Y. Y., Zhang, X. F., Zhong, G. H., et al. (2013). Characterization of a novel cyfluthrin-degrading bacterial strain Brevibacterium aureum and its biochemical degradation pathway. Bioresour. Technol. 132, 16-23. doi: 10.1016/j.biortech.2013.01.002

Chen, S. H., Geng, P., Xiao, Y., and Hu, M. Y. (2012). Bioremediation of $\beta$-cypermethrin and 3-phenoxybenzaldehyde contaminated soils using Streptomyces aureus HP-S-01. Appl. Microbiol. Biotechnol. 94, 505-515. doi: 10.1007/s00253-011-3640-5

Chen, S. H., Hu, M. Y., Liu, J. J., Zhong, G. H., Yang, L., Rizwan-Ul-Haq, M., et al. (2011a). Biodegradation of beta-cypermethrin and 3-phenoxybenzoic acid by a novel Ochrobactrum lupini DG-S-01. J. Hazard. Mater. 187, 433-440. doi: 10.1016/j.jhazmat.2011.01.049

Chen, S. H., Hu, Q. B., Hu, M. Y., Luo, J. J., Weng, Q. F., and Lai, K. P. (2011b). Isolation and characterization of a fungus able to degrade pyrethroids and 3-phenoxybenzaldehyde. Bioresour. Technol. 102, 8110-8116. doi: 10.1016/j. biortech.2011.06.055

Chen, S. H., Lai, K. P., Li, Y. N., Hu, M. Y., Zhang, Y. B., and Zeng, Y. (2011c). Biodegradation of deltamethrin and its hydrolysis product 3phenoxybenzaldehyde by a newly isolated Streptomyces aureus strain HP-S-01. Appl. Microbiol. Biotechnol. 90, 1471-1483. doi: 10.1007/s00253-011-3136-3

Chen, S. H., Yang, L., Hu, M. Y., and Liu, J. J. (2011d). Biodegradation of fenvalerate and 3-phenoxybenzoic acid by novel Stenotrophomonas sp. strain ZS-S-01 and its use in bioremediation of contaminated soils. Appl. Microbiol. Biotechnol. 90, 755-767. doi: 10.1007/s00253-010-3035-z

Corcellas, C., Eljarrat, E., and Barceló, D. (2015). First report of pyrethroid bioaccumulation in wild river fish: a case study in Iberian river basins (Spain). Environ. Int. 75, 110-116. doi: 10.1016/j.envint.2014.11.007

Cycoń, M., Mrozik, A., and Piotrowska-Seget, Z. (2017). Bioaugmentation as a strategy for the remediation of pesticide-polluted soil: a review. Chemosphere 172, 52-71. doi: 10.1016/j.chemosphere.2016.12.129

Cycoń, M., and Piotrowska-Seget, Z. (2016). Pyrethroid-degrading microorganisms and their potential for the bioremediation of contaminated soils: a review. Front. Microbiol. 7:1463. doi: 10.3389/fmicb.2016. 01463

Cycoń, M., Wójcik, M., and Piotrowska-Seget, Z. (2009). Biodegradation of the organophosphorus insecticide diazinon by Serratia sp. and Pseudomonas sp. and their use in bioremediation of contaminated soil. Chemosphere 76, 494-501. doi: 10.1016/j.chemosphere.2009.03.023

Cycoń, M., Żmijowska, A., and Piotrowska-Seget, Z. (2014). Enhancement of deltamethrin degradation by soil bioaugmentation with two different strains of Serratia marcescens. Int. J. Environ. Sci. Technol. 11, 1305-1316. doi: 10.1007/ s13762-013-0322-0

Delgado-Moreno, L., Lin, K., Veiga-Nascimento, R., and Gan, J. (2011). Occurrence and toxicity of three classes of insecticides in water and sediment in two southern California coastal watersheds. J. Agric. Food Chem. 59, 9448-9456. doi: $10.1021 /$ jf202049s

Festa, F., Steel, J., Bian, X., and Labaer, J. (2013). High-throughput cloning and expression library creation for functional proteomics. Proteomics 13, 1381-1399. doi: 10.1002/pmic.201200456

Gao, Y., Chen, S. H., Hu, M. Y., Hu, Q. B., Luo, J. J., and Li, Y. N. (2012). Purification and characterization of a novel chlorpyrifos hydrolase from Cladosporium cladosporioides Hu-01. PLoS One 7:e38137. doi: 10.1371/journal. pone.0038137 $\backslash$ aq $\{11\}\{$ Pleasecite“ Gaoetal.,2012"'insidethetext.

García, M. D., Duque, S. U., Fernández, A. B., Sosa, A., and Fernández-Alba, A. R. (2017). Multiresidue method for trace pesticide analysis in honeybee wax comb by GC-QqQ-MS. Talanta 163, 54-64. doi: 10.1016/j.talanta.2016.10.083

Ghevariya, C. M., Bhatt, J. K., and Dave, B. P. (2011). Enhanced chrysene degradation by halotolerant Achromobacter xylosoxidans using response surface methodology. Bioresour. Technol. 102, 9668-9674. doi: 10.1016/j.biortech.2011. 07.069

Heidari, R., Devonshire, A. L., Campbell, B. E., Dorrian, S. J., Oakeshott, J. G., and Russell, R. J. (2005). Hydrolysis of pyrethroids by carboxylesterases from Lucilia cuprina and Drosophila melanogaster with active sites modified by in vitro mutagenesis. Insect Biochem. Mol. Biol. 35, 597-609. doi: 10.1016/j.ibmb.2005. 02.018

Jilani, S., and Khan, M. A. (2006). Biodegradation of cypermethrin by Pseudomonas in a batch activated sludge process. Int. J. Environ. Sci. Technol. 3, 371-380. doi: $10.1007 /$ BF03325946

Khan, S. U., Bekhi, R. M., Tapping, R. I., and Akhbar, M. H. (1988). Deltamethrin residues in an organic soil under laboratory conditions and its degradation by bacterial strain. J. Agric. Food Chem. 36, 636-638. doi: 10.1021/jf00081a057

Kuivila, K. M., Hladik, M. L., Ingersoll, C. G., Kemble, N. E., Moran, P. W., Calhoun, D. L., et al. (2012). Occurrence and potential sources of pyrethroid insecticides in stream sediments from seven US metropolitan areas. Environ. Sci. Technol. 46, 4297-4303. doi: 10.1021/es2044882

Laskowski, D. A. (2002). Physical and chemical properties of pyrethroids. Rev. Environ. Contam. Toxicol. 174, 49-170. doi: 10.1007/978-1-4757-4260-2_3

Lin, Q. S., Chen, S. H., Hu, M. Y., Rizwan-ul-Haq, M., Yang, L., and Li, H. (2011). Biodegradation of cypermethrin by a newly isolated actinomycetes HU-S-01 from wastewater sludge. Int. J. Environ. Sci. Technol. 8, 45-56. doi: 10.1007/ BF03326194

Liu, J., Chen, S. H., Ding, J., Xiao, Y., Han, H. T., and Zhong, G. H. (2015). Sugarcane bagasse as support for immobilization of Bacillus pumilus HZ-2 and its use in bioremediation of mesotrione-contaminated soils. Appl. Microbiol. Biotechnol. 99, 10839-10851. doi: 10.1007/s00253-015-6935-0

Luong, J. H. (1987). Generalization of monod kinetics for analysis of growth data with substrate inhibition. Biotechnol. Bioeng. 29, 242-248. doi: 10.1002/bit. 260290215

Maloney, S. E., Maule, A., and Smith, A. R. (1988). Microbial transformation of the pyrethroid insecticides: permethrin, deltamethrin, fastac, fenvalerate, and fluvalinate. Appl. Environ. Microbiol. 54, 2874-2876.

Markle, J. C., van Buuren, B. H., Moran, K., and Barefoot, A. C. (2014). "Pyrethroid pesticides in municipal wastewater: a baseline survey of publicly owned treatment works facilities in California in 2013," in Describing the Behavior and Effects of Pesticides in Urban and Agricultural Settings, eds R. L. Jones and S. H. Jackson (Washington, DC: American Chemical Society), 177-194.

Morillo, E., and Villaverde, J. (2017). Advanced technologies for the remediation of pesticide-contaminated soils. Sci. Total Environ. 586, 576-597. doi: 10.1016/ j.scitotenv.2017.02.020

Nagy, K., Rácz, G., Matsumoto, T., Ádány, R., and Ádám, B. (2014). Evaluation of the genotoxicity of the pyrethroid insecticide phenothrin. Mutat. Res. Genet. Toxicol. Environ. Mutagen. 770, 1-5. doi: 10.1016/j.mrgentox.2014. 05.001 
Saikia, N., Das, S. K., Patel, B. K. C., Niwas, R., Singh, A., and Gopal, M. (2005). Biodegradation of beta-cyfluthrin by Pseudomonas stutzeri strain S1. Biodegradation 16, 581-589. doi: 10.1016/j.mrgentox.2014.05.001

Saillenfait, A. M., Ndiaye, D., and Sabaté, J. P. (2015). Pyrethroids: exposure and health effects-an update. Int. J. Hyg. Environ. Health 218, 281-292. doi: 10.1016/ j.ijheh.2015.01.002

Scott, J. G., Yoshimizu, M. H., and Kasai, S. (2015). Pyrethroid resistance in Culex pipiens mosquitoes. Pestic. Biochem. Phys. 120, 68-76. doi: 10.1016/j.pestbp. 2014.12.018

Song, H., Zhou, Z., Liu, Y., Deng, S., and Xu, H. (2015). Kinetics and mechanism of fenpropathrin biodegradation by a newly isolated Pseudomonas aeruginosa sp. strain JQ-41. Curr. Microbiol. 71, 326-332. doi: 10.1007/s00284-015-0852-4

Stok, J. E., Huang, H. Z., Jones, P. D., Wheelock, C. E., Morisseau, C., and Hammock, B. D. (2004). Identification, expression, and purification of a pyrethroid-hydrolyzing carboxylester from mouse liver microsomes. J. Biol. Chem. 279, 29863-29869. doi: 10.1074/jbc.M403673200

Tallur, P. N., Megadi, V. B., and Ninnekar, H. Z. (2008). Biodegradation of cypermethrin by Micrococcus sp. strain CPN 1. Biodegradation 28, 77-82. doi: 10.1007/s10532-007-9116-8

Tang, A. X., Liu, H., Liu, Y. Y., Li, Q. Y., and Qing, Y. M. (2017). Purification and characterization of a novel $\beta$-cypermethrin-degrading aminopeptidase from Pseudomonas aeruginosa GF31. J. Agric. Food Chem. 65, 9412-9418. doi: 10.1021/acs.jafc.7b03288

Topp, E., and Akhtar, M. H. (1991). Identification and characterization of a Pseudomonas strain capable of metabolizing phenoxybenzoates. Appl. Environ. Microbiol. 57, 1294-1300.

Wang, B. Z., Guo, P., Hang, B. J., Li, L., He, J., and Li, S. P. (2009). Cloning of a novel pyrethroid-hydrolyzing carboxylesterase gene from Sphingobium sp. strain JZ-1 and characterization of the gene product. Appl. Environ. Microbiol. 75, 5496-5500. doi: 10.1128/AEM.01298-09

Xiao, Y., Chen, S. H., Gao, Y. Q., Hu, W., Hu, M. Y., and Zhong, G. H. (2015). Isolation of a novel beta-cypermethrin degrading strain Bacillus subtilis BSF01 and its biodegradation pathway. Appl. Microbiol. Biotechnol. 99, 2849-2859. doi: 10.1007/s00253-014-6164-y
Yang, L., Chen, S., Hu, M., Hao, W., Geng, P., and Zhang, Y. B. (2011). Biodegradation of carbofuran by Pichia anomala strain HQ-C-01 and its application for bioremediation of contaminated soils. Biol. Fertil. Soils 47, 917-923. doi: 10.1007/s00374-011-0602-0

Zhai, Y., Li, K., Song, J., Shi, Y., and Yan, Y. (2012). Molecular cloning, purification and biochemical characterization of a novel pyrethroidhydrolyzing carboxylesterase gene from Ochrobactrum anthropi YZ-1. J. Hazard. Mater. 221-222, 206-212. doi: 10.1016/j.jhazmat.2012.04.031

Zhan, H., Feng, Y. M., Fan, X. H., and Chen, S. H. (2018a). Recent advances in glyphosate biodegradation. Appl. Microbiol. Biotechnol. doi: 10.1007/s00253018-9035-0 [Epub ahead of print].

Zhan, H., Wang, H. S., Liao, L. S., Feng, Y. M., Fan, X. H., Zhang, L. H., et al. (2018b). Kinetics and novel degradation pathway of permethrin in Acinetobacter baumannii ZH-14. Front. Microbiol. 9:98. doi: 10.3389/fmicb. 2018.00098

Zhang, C., Wang, S. H., and Yan, Y. C. (2011). Isomerization and biodegradation of beta-cypermethrin by Pseudomonas aeruginosa $\mathrm{CH} 7$ with biosurfactant production. Bioresour. Technol. 102, 7139-7146. doi: 10.1016/j.biortech.2011. 03.086

Zhang, Q., Wang, B. C., Cao, Z. Y., and Yu, Y. L. (2012). Plasmid-mediated bioaugmentation for the degradation of chlorpyrifos in soil. J. Hazard. Mater. 221-222, 178-184. doi: 10.1016/j.jhazmat.2012.04.024

Conflict of Interest Statement: The authors declare that the research was conducted in the absence of any commercial or financial relationships that could be construed as a potential conflict of interest.

Copyright (c) 2018 Yang, Feng, Zhan, Liu, Yang, Zhang, Zhang and Chen. This is an open-access article distributed under the terms of the Creative Commons Attribution License (CC BY). The use, distribution or reproduction in other forums is permitted, provided the original author(s) and the copyright owner are credited and that the original publication in this journal is cited, in accordance with accepted academic practice. No use, distribution or reproduction is permitted which does not comply with these terms. 